\title{
Blockhain Technology As A Me For Sharing Information That Generates User Access Rights and Incentives
}

\author{
Henderi', I Ketut Gunawan², Husni Teja Sukmana ${ }^{3}$, Agung Yudo Ardianto ${ }^{4}$ \\ University of Raharja, Indonesia1,2,4 \\ Syarif Hidayatullah State Islamic University, Jakarta, Indonesia ${ }^{3}$ \\ Jl. Jenderal Sudirman No.40, RT.002/RW.006, Cikokol, Kec. Tangerang, Kota Tangerang, \\ Banten 15117, Indonesia ${ }^{1.2 .4}$ \\ JI. Ir H. Juanda No.95, Cemp. Putih, Kec. Ciputat, Kota Tangerang Selatan, Banten $15412^{3}$ \\ e-mail: henderi@raharja.info ${ }^{1}$, iketut@raharja.info ${ }^{2}$, husniteja@uinjkt.ac.id ${ }^{3}$, \\ agung.yudo@raharja.info4
}

Henderi, Gunawan, I. K. ., Husni Teja Sukmana, \& Ardianto, A. Y. (2021). Blockchain technology as a media for sharing information that generates user access rights and incentives. Blockchain Frontier Technology, 1(01), 01-12. Retrieved from

DOI: https://journal.pandawan.id/b-front/article/view/2

\begin{abstract}
We propose another blockhain client displaying stage that can be utilized to share data without letting completely go and possession and apply it to the field of movement booking. Our new stage gives answers for different central points of interest: extraordinary information insurance and client the board, and shared motivating forces. Track the functioning techniques, objects, time, strategies and reasons for UN associations in an unquestionable way. Gather client profile data and empower clients to trade data with elective travel suppliers to get endowments dependent on protection inclinations determined in great agreements. Client information dependent on the storehouse will be changed over into an open information design and communicated by means of blockchain transmission. This permits the information to be effectively handled and utilized by different hubs. The keen agreement confirms and executes the concurred information. Terms of utilization and token exchange become free help to clients. Savvy agreements can give an assortment of stores to guarantee that all members act with honesty. This record additionally assesses the presentation of the new stage dependent on the investigation of holding up time and memory utilization by characterizing three test situations. The outcomes show that the hub can rapidly react to every one of our issues by utilizing the exchange port non concurrently.
\end{abstract}

Keywords: User modelling, Blockchain, Platform, Intensive.

\section{Introduction}

In recent years, great progress has been made in technological innovation and related research in collaborative methods for exchanging data between companies. Researchsupported data sharing is needed to strike a balance between protecting consumers, improving user experience, and business benefits. There is an increasing discussion and research on what data should be transmitted and how data should be shared to recognize or reward data owners. User data is collected from various parties including companies, including applications, social networking sites, etc., whose main purpose is to improve business models and provide the best services to their consumers. However, storing user data causes serious privacy issues. The 
behaviour comes or will come from a follow-up review of voluntary or observable data. Usually, the ownership specified in the service license agreement refers to the transfer of ownership from the user to the company that obtains the ownership, if shared, through the company network [1].

Data stored in personal vaults may cause privacy and security issues. In this case, even the most popular online platforms face security breaches and data theft. When all data is transferred to a central service provider, centralization issues such as deliberate deletion of user data or the inability to create user data due to technical errors may affect it.

The purpose of exchanging customer information between applications and jobs is to further improve usability, customization of user interfaces and options, and to create a superior customer experience [2] However, when sharing a client, there are problems related to the security, protection, and control of the client. Use standard security policies and scanning methods to solve general security issues, such as: B. Maintain all correspondence without trust, and redefine a common supervisory agency. Various innovations have been achieved, such as B. Computer framework for collecting and providing information, including distributed computer management, RFID (Recognizable Radio Cycle Proof) and various security enhancements to protect customer information collected by programmers. Through unified learning, you can extract information distributed in different areas [3].

However, in order to comply with strict security measures (such as the European Union's GDPR), when submitting information for use by other agencies, it is necessary to obtain the consent of the information owner again. Customers who don't know what to get. Often, the structure of the sentence is long and unclear; they do not provide customers with alternative information that they may share or interfere with other disclosures. It is "with or without life". The customer has not found the answer yet, but bows their heads and clicks "OK", otherwise they will not be able to get any help. As a result, it becomes difficult or even unfamiliar for customers to remember which company they agree with and keep track of who is obtaining their information and for what reason. Obtaining and replacing consent to the use and exchange of information provides customers with sufficient and meaningful incentives, ensuring the exchange of information and ensuring openness to customers, while knowing who received their records and why What is it, such as under what conditions and under what conditions.

We solve all of these issues by providing additional stages to satisfy customers' excellent records and precise agreements, which rely on other customer-oriented methods to protect information-customer safety, security, transparency and control, and The driving force exchange for shared information is coded in a brilliant agreement. It also supports encouraging customers to provide reward information (micro donations or points) encrypted in a smart protocol. In this way, customers will become the owners of their information and can choose how to collect and use their information when transmitting the information. This can be achieved not only by improving the experience that is suitable for providing support, but also directly, for example, by including the advertising revenue of professional cooperatives as a part, we will raise the level of sales execution to a new level. Between various organizations that provide such support in areas such as traffic ordering, information is provided to customers in a decentralized manner. This document is an important continuation of our previous work and calculates the proper execution of the contract [4].In this review article, we used the term "new milestone" to discuss our proposed structure, which uses a different progress record. As required.

The blockchain (advanced logging platform) repository distributes and maintains unchanged hash and encoded information, and any progress or errors can be traced back to the source. From the transaction [5] Multi-chain, as a blockchain, replaces centralized databases with decentralized and released off-chain storage, which can save data storage and bandwidth. In addition, we can store goods distributed offline, thereby saving reservations and transfer rates. However, due to its current technology, MultiChain cannot support the input management components required in the proposal stage, and thus cannot allow customers to indirectly control the way in which their information is distributed and the returns they receive 
[6]. Next, we will use Ethereum. They support excellent transactions and offer barter transactions. Easily access and inspire customers, application owners share customer information. By integrating the security and immutability of the information stored in the blockchain with the special attributes of two famous blockchains (also MultiChain, Ethereum), you can combine their advantages: legal information.

\section{Research Method}

This section provides an introduction to block chain and smart contracts.

\subsection{Blockhain}

If you use Word, use the Microsoft Equation Editor or MathType equation, written in the middle, and given an equation number starting from (1), (2) etc.

\subsection{Literature Review}

Blockchain is an information framework used to make a computerized record dependent on open or private conveyed exchanges that are utilized by a disseminated PC network instead of a solitary supplier. For instance, circulated records can be utilized to follow the possession and status of key resources in the production network. It is repetitively dispersed and put away in the blockchain, and each hub checks each exchange [7]. Accordingly, it is more hard for assailants to assault information and use them for their potential benefit. Therefore, the blockchain is now and then alluded to as "untrusted" in light of the fact that it doesn't need a focal establishment or worker. Record each exchange. Each square gathers numerous exchanges that you need to add to the chain. A cryptographic mark is normally used to recognize each square. Alludes to the mark of the past block in the chain, which can be followed back to the main square in the chain (Genesis).

A definitive arrangement is that no unified authority can decide good and bad. The opposite is likewise obvious, all gatherings rearranged in the arrangement are recorded in the overall record and can be utilized by everybody right now [8]. From a computational perspective, nobody (or dominant part agreement) can change the set of experiences document, on the grounds that the blockchain is the chain of record for the occasion account. Since the data is as yet circulated and excess, each exchange is checked by each hub. Vindictive hubs (degenerate gatherings) can assault and control information for their potential benefit.

Blockchain innovation is relied upon to change the meaning of information the board in different fields [9]. Albeit the blockchain has been created as a stage for virtual cash, its innovation applications are quickly dispersed in different enterprises and money innovation (monetary innovation), just as banking and inventory network businesses. , Many intriguing models utilized in medical care. One of the spaces in which the division at present works is blockchain (appropriated record) innovation as the center of its information the executives framework. In contrast to concentrated frameworks, blockchain innovation is straightforward. Offer information. Obviously, it can likewise help clients share their insight as blessings (micropayments or credits). Encryption in agreement products.

\subsection{Multichain}

MultiChain can be a permissioned private blockchain that can easily provide the required privacy and governance and implement software packages [10]. It is compatible with Windows operating systems and servers, and generates a beautiful JSON-RPC API, which can be directly integrated with existing systems. The main goals of data protection and transparency brought by the integrated user authorization management are three:

1. Only change selected participants to keep the blockchain active,

2. Ensure that only those selected in the transaction area are allowed Specify units,

3. Clearly take out the confirmation of the unprocessed excavation and the associated prices.

MultiChain is a closed system that allows participants to control the maximum block size and set all blockchain parameters in the configuration file to solve scalability issues. It only 
contains transactions that are of interest to participants. The maximum size of chained data on the blockchain is $1 \mathrm{~GB}$, and the information itself is sent quickly through a peer-to-peer (P2P) network. (Initially) the power of all organizations of Genesis employees was mechanically given, including the power to manage users with various access rights [11]. The administrator grants different participants access to network transactions that contain certain data.

\subsection{Ethereum}

Ethereum is a decentralized stage that supports savvy gets, that is, applications that can run freely as arranged without vacation, control, extortion or obstruction from different gatherings [12].

Ethereum utilizes "Ether (ETH)" as a virtual money, which is probably going to be not able to pay for bunch advancements and create a fundamental degree of liquidity for exchanging computerized resources. Ethereum likewise gives an innovation called shrewd agreements. These can be PC codes for mechanical execution of the agreement. Ethereum has "messages" created by outer elements or inside produced by the agreement, which is something contrary to the conduct of Bitcoin bunches that must be produced remotely. There is additionally an unmistakable alternative for Ethereum messages, which contains the message and the beneficiary of the Ethereum message to be answered to. Ethereum likewise has "exchanges"- a marked information bundle putting away messages to be sent from outer records. The exchange incorporates the beneficiary of the message. The mark recognizes the sender, the air volume, and in this manner the transmission data and two qualities, called STARTGAS and GASPRICE [13].

\subsection{Smart Contract}

A shrewd agreement is an arrangement reached between two individuals as PC code. Shrewd agreements are executed on the blockchain network, so they are put away in a public data set and can't be changed. The exchange that happened. They are prepared in keen agreements. This implies that keen agreements can be sent consequently without the association of any outsiders (banks, governments, and so forth) Possibly exchange when the particulars of the arrangement are met. Without an outsider, there is no dependable presence to execute savvy contracts [14].

Brilliant agreements contain a few capacities that can be gotten to from outside the blockchain or through proper elective agreements. Notwithstanding solid agreement innovation, blockchain additionally wipes out the dependence on a focal framework between parties associated with exchanges [15]. Since sensible agreements are kept up on the blockchain, all reports related with parties on the organization have duplicates.

Complete occasion observing log. At the point when one gathering attempts to change the agreement or offer on the blockchain, any remaining gatherings recognize and keep away from the present circumstance. On the off chance that one side comes up short, the framework will keep on working without loss of data or respectability. We have made a sensible ADP framework that is secure, complete and liberated from unified dangers, expenses, and model trust issues [16].

As of now, we just utilize the Solidity 2 programming language to make savvy contracts since it just duplicates the essential activities of the fundamental form, which simplifies the code. EVM code (Ethereum Virtual Machine) 3 is utilized for gets that contain one byte. Every byte addresses an assistance object. The code can get to the Wei code sent in the exchange and the data entered in the message, lock the header data, and return it as a yield to the PC's stockpiling block cluster. The truffle4 system has great inner agreement, restricting, parallel execution and the board capacities, which can be utilized for acceptable agreement arrangement and testing of the EThereum blockchain. Presently, we are as yet utilizing EVM with great agreements. As Ethereum Assembly (EWASM) 5 shows up on the Internet later on, great agreement configuration may require programming dialects other than 
Solidity, which additionally accelerates the call between Web Assembly and JavaScript (JS).

\subsection{The Proposed Platform}

The Data Protection Law and the General Data Protection Regulation (RGPD) of October 25, 2018 regulate the processing of personal data and force the deletion of personal data. However, the information about the regional units of the blockchain remains the same. Blockchain is used as part of the solution, which can be tricky. Therefore, we created a universal file system design for sharing user information that supports blockchain information and offline storage (see Figure 1). Specific information is rarely displayed in the blockchain. User information is hashed and encrypted before being uploaded to offline storage. The application immediately stores information about home buyers in offline storage. The conditions for accessing the user information area block are encoded in the corresponding information, and the data identifies the final contract and is published on the blockchain platform (Ethereum).Blockchain learning prevents middleware from manipulating information. Using content-based addressing, you can characterize the information used as search characters. If the data client requests a reasonable contract to access user information, only the successful contract request will release the key used to decrypt the user information. Then, the final program extracts the hash from the block string, uses the hash to extract the information from the off-chain repository, decrypts and sends the information to the data client, thereby reducing ownership linformation.

Blockchain and smart contracts help users by providing complete visibility into when and where UN agencies access their information so that users can determine the distribution of information. Companies that can access information and encourage AN users to provide their information (related to payment for information related to the use of the application; according to the contract). As a chain mechanism for storing information about users, the mechanism can be in a place where information is stored. You think there is a group of centralized service providers to store and manage all information. This makes it difficult to reduce the risk difference. For example, if a company shuts down without user consent, other organizations will be harassed, hacked, overused or even destroyed.

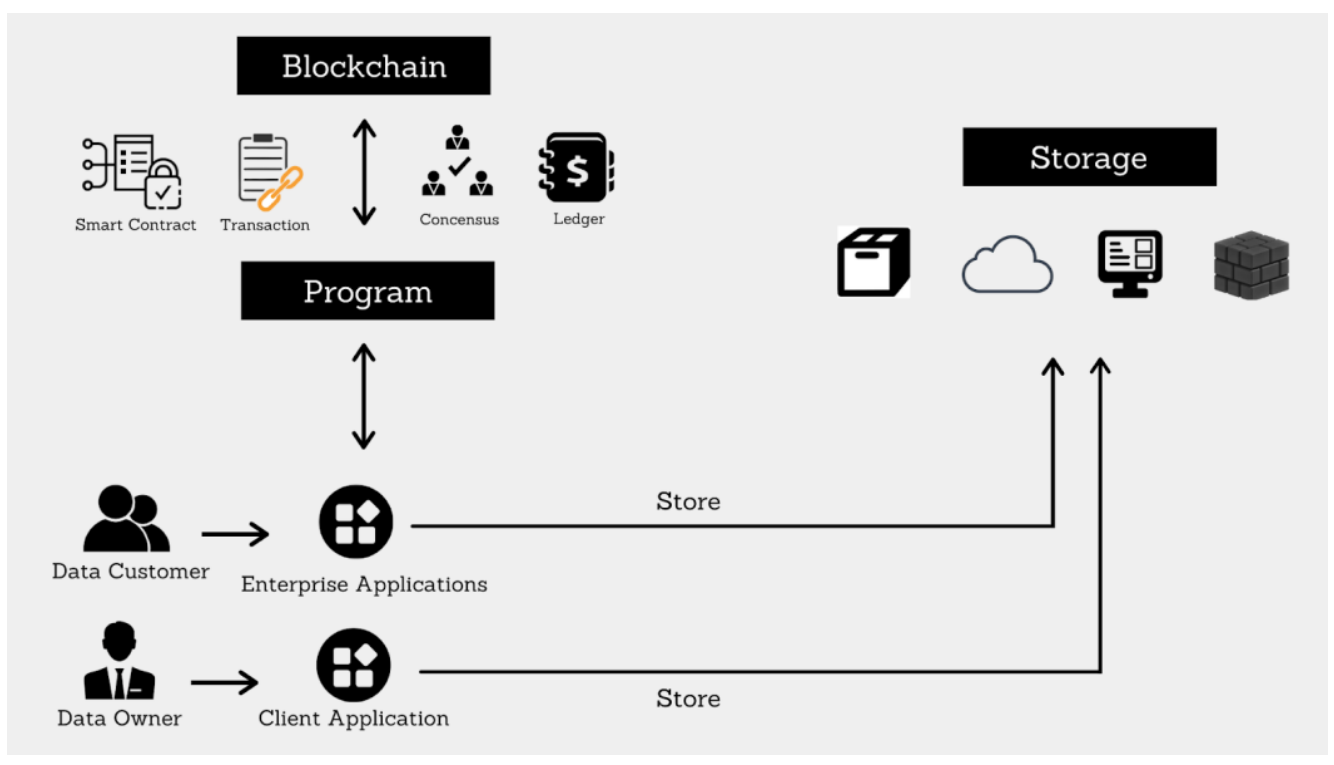

Figure 1. Design For Sharing User Information 
Therefore, we want to provide a separate platform to replace the privately-licensed MultiChain blockchain as a solution for storing, encrypting, hashing, and searching knowledge on the Ethereum side (for access control) inside and outside the chain [17]. A limited number of pairings in MultiChain can be used to successfully implement an off-chain blockchain implementation with stored user information. Users can also save the printed information offline, thereby saving storage space and information size. Off-chain and access through MultiChain are also in our previous analysis work and other work such as Rule. Ferrer-Sapena and Sanchez- Parez [18] have been planned. MultiChain nodes perform important operations, such as: B. Hashing and encrypting user data, storing encrypted files in the country (outside the blockchain), and hashing the files on the blockchain to display the required information, Verify the information and transmit the information [19].

Therefore, we introduced two blockchains: MultiChain, which is used to exchange user profile data between companies on private networks and Ethereum, to maintain robust access control policies (such as reasonable contracts). The user logs in by providing his basic profile data and activating the smart contract. The associated network node Ethereum Ethereum node can automatically realize the user experience to maintain user-controlled privacy in the following aspects: (a) share the user's knowledge with it; and (b) once its knowledge is transferred to an alternative third party, the user Will still be motivated. Communication with the nodes of the Ethereum network is carried out directly through our own company nodes. Although the confidential contract of Ethereum will continue, users will have their own Ethereum addresses. After users learn about other participating organizations and use related users (provider data), they can safely store Ethereum (ETH) in their In your own refrigerated container (offline wallet). Irritated by ether. We tend to justify the workflow in the section "Shared User Incentives" [20].

In order to exchange user information between companies, the MultiChain blockchain is hosted at each location of participating companies, for example B. Under a series of reasonable contracts, publish content on the Internet and share them online by user name.

\section{Method}

We plan and execute a common reinforcement organization to construct applications on UNIX PCs. Web applications are created utilizing PHP and Apache and MySQL workers as backends. The PC running MultiChain is one on all hubs and gathers data from the customer through right confirmation. The Ethereum blockchain local area stores and executes significant agreements. Every single enrolled client and client data have an Ethereum address. At the point when information is traded by a sensible agreement, this square size won't send ether. The client makes his profile in the structure reservation framework. Since the underlying (hub 1) in the blockchain document recorded its data, and simultaneously chose to impart data to an outsider. The data put away in the vault will be changed over into open information, which will be planned to JSON design and printed through the stream in MultiChain. The stream in MultiChain is utilized for general data stockpiling. Outsiders, for example, Saskatoon Travel and Tours and Saskatoon Mall have totally various centers, and the arrangement of these center points additionally incorporates Multichain. You will get a location and a few licenses to remain on the shut blockchain network. A hub has numerous addresses, and these addresses play out quite a few exchanges between them, which further anonymizes the interaction. Consequently, all associated hubs will create their own public location and private key pair. At the point when two associated blockchain hubs are constrained by the accompanying calculation A [22], 
MultiChain confines a bunch of permitted clients to get to the blockchain and adds a "handshake" technique.

\section{STEP A.}

1. Node is known as public address.

2. A node verifies that someone else's address is on its own version of the whitelist.

3. The node sends a challenge message to the opposing party.

4. The node sends back the signature of the challenge message to prove ownership of the private key linked to the public public address they provide. If any node is not happy with the result, it cancels P2P affiliation.

MultiChain restricts access to the blockchain by multiple users with permissions, and adds a "handshake" method that uses the following algorithm $A$ to manage two connected blockchain nodes. As shown in Figure 3, we usually run Multichain to provide building security for the master node in the building. (Node 1) is in the blockchain. This node has the role of assigning administrator and grants the assigned access authority of the standby node [23]. In our example, the authority of the replacement node of the area unit is set by the master control node, and is set to true for all nodes when adjusting the chain parameters. The main parameters of the network installed in our multi-chain area are as follows

1. chain-protocol = multichain \# Chain protocol

2.chain-description = MultiChain Model \# Chain Description

3. root-stream-name = root \# Root stream name

4. root-stream-open = true \# allows anyone to publish root streams

5. chain-is-testnet = false \# Fill in the "testnet" field in the response API, for compatibility.

Base chain parameters are set to limit the permissions of each new leap node. Likewise, worldwide permissions in the multichain area unit file as shown below: Permissions \#Global

1. anyone-can-connect = false \# non-public blockchain.

2. anyone-can-send = false \# group action language is not restricted by address.

3. anyone-can-accept $=$ false \# output action group unit area delimited by address.

4. anyone-can-accept-empty = true \#without grant permission, transfer quality and nil \$

5. anyone-can-create = false \# selected will generate a new stream.

6. anyone-can-issue $=$ false \# selected will publish the new original assets.

7. anyone-can-mine = false \# selected will mine the block (confirm transaction).

8. anyone-can-activate = false \# selected will grant or revoke the link, send and accept permissions.

9. Anyone-can-admin = false \# that is selected will grant or revoke all permissions.

10.support-miner-precheck $=$ true $\#$ Requires output specific metadata with cached scriptPubKey for input, to support my advanced \$

This makes the Multi Chain Core Daemon determine which different nodes can connect to with this node exploit the command:

multi chain model @ [ip]: [port] (e.g., multi chain model@192.168.204.132: 8353)

User Incentives for Sharing

We execute smart contracts in Ethereum to ensure that users can be confident after participating company nodes use user information. According to the appropriate contract [24], all users (data providers) and nodes of participating companies (data consumers) have an 
Ethereum mobile address. The user decides which customer (application or company) will access their data. The implementation here stipulates that the user agrees to the terms that allow the company to obtain permission to collect and use content before using its service, and states that the World Health Organization users share the information that retains their content, but the company website also agrees to the ongoing operation. License restrictions (and the right to sub-license) to distribute such content (see Appendix A and Appendix B for the terms of the agreement). Users who access their information as long as the World Health Organization once and for any purpose [25].

\section{STEP B}

Inputs: Acu, Aco, Acc, deposit, dataPrice, contractState

1. Acu, Aco, unit area commands the set of all consumer ether addresses, customer knowledge

and contracts, separately.

2. Give access only to the Acu $\varepsilon$ Acu, Aco $\varepsilon$ Aco the World Health Organization that are registered into the system.

3. Change the contract status to be Created.

4. deposit reference e d.

5. Set the knowledge score to the specified ep ep $=1 / 2 \mathrm{e}$.

6. The balance order contract is metal = dysfunction, wherever the command order A cc.

7. Aco permission to determine the client's knowledge of his interests.

\section{STEP C}

Inputs: Acu, Aco, Acc, deposit, dataPrice, contractState

1. Acu, Aco, an order is a collection of all the buyer, buyer, and ETH addresses

contract, separately.

2. Aco decide to consume acu client information, pay the 2ep

consumer savings $=e \mathrm{p}$.

3. The balance of the order contract is metal element $=E D+$ two

* e p.

4. modification of contract status for safekeeping.

5. Provide Aco to access client information.

6. Aco confirms the availability of information

7. Change the contract status to Inactive.

8. Transfer ep deposit from command back to co.

9. The remainder of the Contract balance orders to be metallic elements $=E D+e p$.

10. Transferring metallic elements to atomic number 29.

Initially, only some companies could access user information by subscribing to programs published on MultiChain. By broadcasting Ether to the user's broadcast address, incentives are provided for various digital tokens [26].

Because of Ropsten, we utilize the Ropsten blockchain to execute the Remix IDE12 contract on the web, which might be a check network with a comparable PoW (Proof of Labor) 
component to confirm the square, very much like in the principle Ethereum organization, and Remix can give an IDE to allowed to connect untrusted code before it is delivered. Use Etherscan 13 to explore the Robsten blockchain and check each area of exchanges executed on the blockchain. Likewise, we normally use metamask14 to execute Web3 setup records, which are presented for adding contracts with Ethernet account addresses. Figure 7 shows a square chart of the work process rationale. Complete approach cycle, and draft agreements to deal with the principle expenses of information sharing.

We limit the capacities (strategies) to significant agreements, contingent upon the jobs of the members in question, to perform various undertakings between the data supplier and the client in arrangement. The means to acquire the region code are depicted in Rule B and Algorithm C. Factors contain Ethereum address, cost data and agreement status. On the off chance that vital, we will in general create an advantage setting system to produce an option in contrast to the situation with the parent contract or the youngster contract. Thusly, the compiler will naturally create get capacities for every open variable.

\section{- Experimental Setup 1}

In order to achieve the three main goals, the analysis focused on three cases to simulate very different degrees of parallelism while monitoring the latency on Windows and UNIX computers. Table 1 shows the 3 units of the situation area. We tend to suspend the process further. If key operating system processes are not running with the multi-chain daemon in the background to confirm that there is no other way, then this will affect our experiments. These experiments were performed on the newly created Multichain node. Because MultiChain uses an encryption mechanism, block indexing and link access are limited to users who are whitelisted. This is how we create blockchain nodes like modern nodes. The block index is the information stored for each block, no matter where it is stored on the hard disk. In the status bar, the data about the subsequent verification status will be saved as the result of the current known string. As mentioned above, the node used to store the key-value pairs and hash state of all blocks [27].

The theoretical maximum amount of information for network membership depends on the technology used.However, the actual number of packets sent over the network depends on the higher latency and the lower latency. Excessive delay will prevent information from filling the network pipeline, reduce throughput and limit the most effective information size of the connection. We strive to adjust our analysis in a way that considers latency and memory consumption in every situation.

To observe the results of the multi-chain core daemon stopping and reconnecting to the network, we tend to create a script that runs with a one minute pause for each new observation with the following Multichain command:

1. multichain-cli model stop

2. multichaind model daemon

We ensure the latency of the primary node Node one, once obtained connect to a different single N2 node for S1 business, next when it is connected to 2 nodes opposite N2 and N3 for S2 state and equal to seven different nodes N2-N7 for state S3 in a total of twenty observations.

For S3, we tend to record the first latency of Node one to install it with seven opposite nodes in the network and finally average them to find the average latency for connecting seven very different nodes from Node1.

Additionally, we have a tendency to give another experiment to observe the memory consumption for a node after the corresponding multichain core daemon is started on it a particular node. The full range of 5 observations has been shared, one of which is shown in Figure eight, 9. The figure shows the total memory usage during pre and post Daemon multichainactivation. 


\section{- Experimental Setup 2}

To accomplish the last objective, the examination is identified with executing the code with Remix IDE on the Ethereum Ropsten testnet. as of not long ago, a reasonable agreement required some fuel to store code and complete exchanges onto the Ethereum blockchain, and its actual worth was paid in ether. SPBU ETH15 gives 3 gas cost classifications. they are SafeLow ( $<30 \mathrm{~min})$, Standard ( $<5 \mathrm{~min})$, and quick $(<2 \mathrm{~min})$. As far as possible is helpful for advancing the gas utilized offers a security system, as a rule code with bugs may stay with that extreme gas save for execution. We tend to utilize the gas worth of 25 Gwei (2.5e-8 ether) that is the worth to accomplish quicker exchanges. esteem a The exchange will keep on expanding after the gas esteem gets higher. We give a one-in-one illustration of making contracts throughout the long term paper with the following division hash:

H1: 0xe7b67820af62d3dc5c41357a6de1192de14f8c39cc0416a2830c57c28e3c32b6

H2: 0x8b38cc9b56f584ccec022678f61b16b166e32e581fd0329a394599000af8f226

H3: 0xd333d232646a571be438cda52548503f07047fa07dcce026f18b8df2c88870d0

For this situation, an agreement is first made at the location AN "0x2aadf80E4CE7Fc2Db5d57dD975e0337D373e1C50" with H1 hash business. From that point onward, the two ether are moved to the agreement from the customer, which is at the location AN "0x5378fa11529725ccc491bb6708f9e2f06a1639d5." The value information is characterized as one ether. In this way, the customer should get three meters toward the finish of the exchange. customer data at the location AN "0x923c1eDfAdB6332254C83 BCbAE85B2cA6b9Bb36e" with $\mathrm{H} 2$ hash exchanges move two ether to get which ether is a store. At last, the customer gets the data record client information, and the agreement moves one ether to information customer and three ether to customer by exchange.

\section{Results And Discussion}

Information on inertness for the principle a piece of the perception are appeared in Table two and Figure eleven. All circumstances have a base and probably an idleness of roughly hundred and fifty $\mathrm{ms}$, independently, giving an average dormancy season of $<125$ ms.

Generally speaking there is no quantifiable breaking point in the quantity of hubs as every hub doesn't need to be associated with one another to make a completely associated shared organization on a private blockchain. In addition, prior to testing basic machines, we would in general have performed comparative tests at first on different virtual machines, and we found that our outcomes were comparative in square size for each situation. the solitary distinction here with real actual hubs is that they mirror the basic state with no organization issues influencing the investigation. Be that as it may, for all hub make up for lost time, the new hub association should circle back all exchanges from the beginning chain, at that point it will require some investment prior to refreshing. the specific measure of time can rely upon what square of numbers and exchanges are in the chain.

Our investigations have been completed with just ten streams having a sum of 100 things, that is under $100 \mathrm{MB}$. that is because of We tend to just draw in with idleness. moreover, since no sensible agreement was made to Multichain, not at all like in Ethereum, there isn't any execution driven by the program motor \{for each | for each\} message on each blockchain hub. That certainly adds to the low dormancy we are probably going to affirm here.

We additionally broke down the memory utilization for multichain Node documents after their center daemon was begun. We mentioned twenty successive objective facts to date to envision the memory utilization, which was adjusted from the underlying memory use of 938 to $970 \mathrm{MB}$ when the multichain daemon was begun. In this way, it would be the general memory use that was around $28 \mathrm{MB}$ and it would not be too large to work with a model with 
a multichain Blockchain record. In addition, it additionally upholds the amount of exchanges that are not wrapped up.

There are likewise around 300 bytes of memory put away for each square in the chain. In this way, assuming the hub is bought in to innumerable streams, it might expand memory utilization. Be that as it may, our model focuses on the capacity of client profile data, and surprisingly $1,000,000$ of this data can have a size of just around $100 \mathrm{MB}$. Thus, this model is very viable as far as quick beginning, quick reaction, and less memory utilization.

Further, we tend to dissect the worth of gathering activity while sending conceivable agreements and capital punishment related capacities. As will be found in Figure twelve, for instance, the cost to be executed for settlement of installments (ether move of the agreement applied to customers and purchasers) is 0.001247 ether once the upper gas costs for the more quickly chose bunch activity. This worth is 0.18 USD and is considered worthy according to the expense of value gas for Ethereum 17 All elements of the Contract were tried effectively with different restriction jobs. Table three presents the expense and time bunch activities utilized for different capacities (counting constructors) as shifted as agreement states. the full worth related with the agreement is 0.022979 ether, that is, the expansion of the death penalty cost of various constructors and capacities. the cost for executing perform to change the agreement status from "Bolted" to "Idle" is higher than that for differing the status from "Made" to "Bolted". This expansion is even because of the past approach which made the agreement move ether to every customer and customer data, yet then made the customer's information move ether to the shrewd agreement.

Subsequently, we will in general assess the exhibition of our new stage by executing it under an information sharing model and carrying out it under a client motivating force model. The outcomes show that our data sharing model has low inertness for appending multichain hubs to desperately associate with the organization and to react to activities like beginning a stream, seeing a thing stream, stacking or distributing those things to the stream. Likewise burns-through less memory after the blockchain daemon is begun. The client motivating force model has the fitting gathering activity an incentive for executing a reasonable agreement.

Subsequently, this new stage upholds blockchain and bodes well the agreement innovation permits the development of programmed confirmation of conditions for access or change of any data substance. The brilliant agreement will be utilized to write in the allowed work code of the utilization of information, the allowed use of the product bundle, the UN office individual or business will get to the data, time limits, access esteems, and so on Accordingly, this new rearranged information sharing stage is valuable for sharing client data of different kinds (client model and information client commitment), by giving answers for security, client the board and motivator issues. This usable blockchain-based stage will permit clients to make an image of possession and spot of beginning of their information, share data without losing that administration and proprietorship, give/get motivations to share and give clients full straightforwardness and the board of UN organizations getting to their data, when and for what reason. Be that as it may, the main analysis for blockchain-based ways to deal with this point identifies with their presentation and adaptability for the overall population Ethereum blockchain; Nevertheless the fast advancement of innovation made it conceivable, through savvy blockchain mix to accomplish adequate execution.

\section{Conclusion}

To sum up, we directed a trial investigation of our most recent blockchain-based system for sharing client profile information, which permits clients to keep up power over shares while procuring rewards. It is centered around savvy gets that incorporate client controlled protection and information sharing arrangements. This likewise normally upholds the improvement of motivations for clients to share their profile information as remunerations 
(miniature installments or credit). Therefore, purchasers become information proprietors, with the capacity to control how their information is put away, utilized, and traded. The stream definition from Multichain has been effectively deciphered by taking the instance of the movement booking area to trade client profile information in a circulated way. As one of the Multi Chain endeavor hubs, we present a lodging reservation framework that gathers client profile information and permits clients to get motivators while trading their profile information with other travel ventures dependent on their security inclinations expressed in brilliant agreements. The vault client information is converted into open information design and traded through the stream on the blockchain so different hubs can measure and utilize it effectively. Shrewd agreements confirm and authorize concurred information use terms and move computerized tokens to information proprietors as motivating forces. To guarantee that all members act truly, savvy contracts require a twofold store ensure. This paper has introduced the central employments of savvy contracts for security keeping up information sharing and the board. We joined blockchain and off-anchor vaults to make an information sharing and the executives model that focuses on security and protection. This blockchain-empowered client information sharing model applies not exclusively to the movement area, yet in addition to other related areas like eCommerce, schooling and wellbeing. This paper additionally evaluates the achievement of our new stage, which surpasses our principles as far as execution.

\section{Acknowledgment}

With high enthusiasm and motivation to work on a journal entitled "Blockchain Technology as a Media for Sharing Information that Generates User Access Rights and Incentives" Thank you to Raharja University and also Alphabet Incubator who have fully supported this research. So that participation in this research is able to expand knowledge for myself and for other communities, especially for the world of digitization.

\section{References}

[1] K. M. Moriarty, Transforming Information Security: Optimizing Five Concurrent Trends to Reduce Resource Drain. Emerald Group Publishing, 2020.

[2] U. Rahardja, Q. Aini, H. D. Ariessanti, and A. Khoirunisa, "Pengaruh Gamifikasi pada iDu (iLearning Education) dalam Meningkatkan Motivasi Belajar Mahasiswa," NJCA (Nusantara J. Comput. Its Appl., vol. 3, no. 2, pp. 120-124, 2018.

[3] U. Rahardja, I. Handayani, and A. A. Ningrum, "Pemanfaatan Sistem iMe Berbasis WordPress sebagai Official Site RCEP pada Perguruan Tinggi," Creat. Inf. Technol. J., vol. 4, no. 3, pp. 207-219, 2018.

[4] S. Ayvaz and S. C. Cetin, "Witness of Things: Blockchain-based distributed decision record-keeping system for autonomous vehicles," Int. J. Intell. Unmanned Syst., 2019.

[5] Q. Aini, Y. I. Graha, and S. R. Zuliana, "Penerapan Absensi QRCode Mahasiswa Bimbingan Belajar pada Website berbasis YII Framework," Sisfotenika, vol. 7, no. 2, pp. 207-218, 2017.

[6] N. Siegfried, T. Rosenthal, and A. Benlian, "Blockchain and the Industrial Internet of Things: A requirement taxonomy and systematic fit analysis," J. Enterp. Inf. Manag., 2020.

[7] A. B. Turner, S. McCombie, and A. J. Uhlmann, "A target-centric intelligence approach to WannaCry 2.0," J. Money Laund. Control, 2019.

[8] J. Lin, Z. Shen, C. Miao, and S. Liu, "Using blockchain to build trusted LoRaWAN sharing server," Int. J. Crowd Sci., 2017.

[9] D. Sinha and S. R. Chowdhury, "Blockchain-based smart contract for international business-a framework," J. Glob. Oper. Strateg. Sourc., 2021.

[10] R. L. Rana, C. Tricase, and L. De Cesare, "Blockchain technology for a sustainable agri- 
food supply chain," Br. Food J., 2021.

[11] P. R. Sousa, J. S. Resende, R. Martins, and L. Antunes, "The case for blockchain in loT identity management," J. Enterp. Inf. Manag., 2020.

[12] N. Kant, "Blockchain: a strategic resource to attain and sustain competitive advantage," Int. J. Innov. Sci., 2021.

[13] S.-C. Oh, M.-S. Kim, Y. Park, G.-T. Roh, and C.-W. Lee, "Implementation of blockchainbased energy trading system," Asia Pacific J. Innov. Entrep., 2017.

[14] R. P. George, B. L. Peterson, O. Yaros, D. L. Beam, J. M. Dibbell, and R. C. Moore, "Blockchain for business," J. Invest. Compliance, 2019.

[15] Q. Aini, I. Handayani, and C. A. Seto, "Content Management System Zpreneur in Support of Entrepreneurship llearning at Perguruan Tinggi Raharja," Creat. Commun. Innov. Technol. J., vol. 8, no. 3, pp. 233-245, 2015.

[16] U. Rahardja, Q. Aini, and D. Sartika, "Build A Business To Customer Online Store Using Airzone Content Management System," CCIT J., vol. 8, no. 2, pp. 112-122, 2015.

[17] U. Rahardja, I. Handayani, and B. A. Pahad, "Pemanfaatan Rinfoform Sebagai Media Request Update Artikel Pada Iran," CSRID (Computer Sci. Res. Its Dev. Journal), vol. 8, no. 3, pp. 191-200, 2016.

[18] X. Xue, J. Dou, and Y. Shang, "Blockchain-driven supply chain decentralized operations-information sharing perspective," Bus. Process Manag. J., 2020.

[19] P. Xie, Q. Chen, P. Qu, J. Fan, and Z. Tang, "Research on financial platform of railway freight supply chain based on blockchain," Smart Resilient Transp., 2020.

[20] U. Rahardja, Q. Aini, and A. Khoirunisa, "The Effect of Rinfogroups as a Discussion Media in Student Learning Motivation," Aptisi Trans. Manag., vol. 2, no. 1, pp. 79-88, 2018. 